\title{
Gift, honorary or guest authorship
}

LA Harvey ${ }^{1}$

Published online: 9 February 2018

(C) International Spinal Cord Society 2018

The pressure on researchers to publish encourages them to put their names to papers that they may not have contributed to in a meaningful way, and could not possibly defend if the need arose [1]. Interested readers are directed to a poignant paper written on this topic in 2003 [2]. This paper tells of two well-known cases of scientific fraud in the 1980s by Darsee and Slutsky. However, of particular interest was that:

"...the co-authors who had been willing to accept credit for work they had not done proved unwilling to share responsibility for the fraudulent papers" (page 244) [2].

At the time when this issue arose, there was much discussion about what constitutes authorship. Leaders in the field argued that authorship required:

"meaningful participation in the planning, design, and interpretation of the experiments and in the writing of the paper." (page 1417) [3].

And that:

"anyone who allows his or her name to appear among the authors of a paper assumes major responsibilities.... Co-authors should be able and willing to defend the paper in public, and that means they must be confident about the integrity of the data. Furthermore, coauthorship should never be conferred or accepted as an honor or simply as a reward for providing resources or sponsoring a junior investigator who has done all the work. (p.1417) [3].

The discussion around this topic at the time led to the introduction of the terms gift, honorary, and guest authorship. Gift authorship refers to the practice of offering authorship to a senior or junior colleague in the blatant or surreptitious hope that they will return the favor. Honorary authorship refers to those who are named as authors merely because they hold senior positions within the service or facility where the research occurred, and may have helped secure funding. Guest authorship refers to senior authors who are included because of their respect or influence in the hope that this will increase the likelihood of publication and/or impact of the paper once published. All three types of authorship are at best opportunistic and at worse scientific misconduct [4].

Spinal Cord heeds the advice of the International Committee of Medical Journal Editors on authorship, and strongly discourages gift, honorary or guest authorship. Spinal Cord encourages its authors to follow the authorship guidelines recommended by the International Committee of Medical Journal Editors [5]. They state that authorship is justified when individuals satisfy the following 4 criteria:

\section{LA Harvey}

spinalcord@iscos.org.uk

1 University of Sydney, Sydney, Australia
- Substantial contributions to the conception or design of the work; or the acquisition, analysis, or interpretation of data for the work; AND

- Drafting the work or revising it critically for important intellectual content; AND

- Final approval of the version to be published; AND

- Agreement to be accountable for all aspects of the work in ensuring that questions related to the accuracy or integrity of any part of the work are appropriately investigated and resolved.

The issue is however less clear for papers that do not involve original research but instead summarise opinions, provide recommendations or interpret evidence. Typically these types of papers have an extensive list of authors and come in the form of practice guidelines, consensus papers or position statements. The strength and appeal of these papers are the involvement of so many respected experts in the field. This gives the paper authority. But sometimes the respected experts have only attended a workshop and/or perhaps provided comments on a draft of the paper. Does this warrant authorship? The 4-point criteria for authorship provided by the International Committee of Medical Journal Editors provides little guidance because it is less relevant for these types of paper. Yet common sense suggests that perhaps instead of long lists of authors for these types of papers, there should be a statement at the end of the paper outlining those who endorse the recommendations of the paper and/or contributed to the paper in a minor way, and authorship should be reserved to those who did the leg-work and made important contributions to the paper. I will leave all to ponder this contentious issue.

Spinal Cord discourages gift, honorary, or guest authorship. It is the responsibility of lead authors of papers to ensure all authors contribute to their papers in a meaningful way. To increase transparency around this issue, Spinal Cord now requires authors to add a section to the end of their manuscripts which clearly articulates the contribution of each author.

\section{References}

1. Gasparyan AY, Ayvazyan L, Kitas GD. Authorship problems in scholarly journals: Considerations for authors, peer reviewers and editors. Rheumatol Int. 2013;33:277-84.

2. Jones AH. Can authorship policies help prevent scientific misconduct? What role for scientific societies? Sci Eng Ethics. 2003;9:243-56.

3. Relman AS. Lessons from the Darsee Affair. N Engl J Med. 1983;308:1415-7.

4. Rajasekaran S, Li Pi, Shan R, Finnoff JT. Honorary authorship: frequency and associated factors in physical medicine and rehabilitation research articles. Arch Phys Med Rehabil. 2014;95:418-28.

5. ICMJE. Defining the Role of Authors and Contributors. 2018. http://www.icmje.org/recommendations/browse/roles-andresponsibilities/defining-the-role-of-authors-and-contributors.html. 